ISSN 1855-3966 (printed edn.), ISSN 1855-3974 (electronic edn.)

ARS MATHEMATICA CONTEMPORANEA 20 (2021) 29-36

https://doi.org/10.26493/1855-3974.2338.5df

(Also available at http://amc-journal.eu)

\title{
A family of fractal non-contracting weakly branch groups
}

\author{
Marialaura Noce * \\ Georg-August-Universität Göttingen, Mathematisches Institut
}

Received 20 May 2020, accepted 21 July 2020, published online 13 July 2021

\begin{abstract}
We construct a new example of an infinite family of groups acting on a $d$-adic tree, with $d \geq 2$ that is non-contracting and weakly regular branch over the derived subgroup.
\end{abstract}

Keywords: Groups of automorphisms of rooted trees, branch groups.

Math. Subj. Class. (2020): 20E08

\section{Introduction}

Weakly branch groups were first defined by Grigorchuk in 1997 as a generalization of the famous $p$-groups constructed by Grigorchuk himself [4, 5], and Gupta and Sidki [6]. These groups possess remarkable and exotic properties. For instance, the Grigorchuk group is the first example of a group of intermediate word growth, and amenable but not elementary amenable. Also, together with the Grigorchuk group, other subgroups of the group of automorphisms of rooted trees like the Gupta-Sidki $p$-groups and many groups in the family of the so-called Grigorchuk-Gupta-Sidki groups have been shown to be a counterexample to the General Burnside Problem.

For these reasons, (weakly) branch groups spread great interest among group theorists, who have actively investigated further properties of these in the recent years: justinfiniteness, fractalness, maximal subgroups, or contraction.

Roughly speaking, a group is said to be contracting if the sections of every element are "shorter" than the element itself, provided the element does not belong to a fixed finite set, called the nucleus (see the exact definition in Section 2).

* The author wants to thank Laurent Bartholdi for pointing out the existence of [9], and Gustavo A. FernándezAlcober and Albert Garreta for useful discussions. The author thanks the anonymous referee for helpful comments. The author is supported by EPSRC (grant number 1652316), and partially by the Spanish Government, grant MTM2017-86802-P, partly with FEDER funds.

E-mail address: mnoce@ unisa.it (Marialaura Noce) 
Even though in the literature there are many examples of weakly branch contracting groups, not much is known about weakly branch groups that are non-contracting. In 2005 Dahmani [2] provided the first example of a non-contracting weakly regular branch automaton group. Another example with similar properties was constructed by Mamaghani in 2011 [7]. Both are examples of groups acting on the binary tree. We also point out that in [9] Sidki and Wilson proved in particular that every group acting on the binary tree with finite abelianization (including non-contracting groups) embeds in a branch group. This provides more examples of non-contracting branch groups acting on the binary tree.

For $d \geq 3$, the Hanoi Towers group $\mathcal{H}(d) \leq$ Aut $\mathcal{T}_{d}$ (which represents the famous game of Hanoi Towers on $d$ pegs) is non-contracting and only weakly branch. To the best of our knowledge if $d>3$ it is not known if these groups can be branch. For more information on the topic, see [3] and [10].

In this paper we explicitly construct an example of an infinite family of non-contracting weakly branch groups acting on $d$-adic trees for any $d \geq 2$. This result gives a wealth of examples of groups with these properties. In the following we denote with Aut $\mathcal{T}_{d}$ the group of automorphisms of a $d$-adic tree.

Theorem 1.1. For any $d \geq 2$, there exists a group $\mathcal{M}(d) \leq$ Aut $\mathcal{T}_{d}$ that is weakly regular branch over its derived subgroup, non-contracting and fractal.

\subsection{Organization}

In Section 2 we give some definitions of groups acting on regular rooted trees and of properties like fractalness, branchness and contraction. In Section 3 we introduce these groups and we prove the main theorem together with some additional results regarding the order of elements of $\mathcal{M}(d)$.

\section{Preliminaries}

In this section we fix some terminology regarding groups of automorphisms of $d$-adic (rooted) trees. For further information on the topic, see [1] or [8].

Let $d$ be a positive integer, and $\mathcal{T}_{d}$ the $d$-adic tree. We denote with Aut $\mathcal{T}_{d}$ the group of automorphisms of $\mathcal{T}_{d}$. We let $\mathcal{L}_{n}$ be the $n$th level of $\mathcal{T}_{d}$, and $\mathcal{L}_{\geq n}$ the levels of the tree from level $n$ and below.

The stabilizer of a vertex $u$ of the tree is denoted by st $(u)$, and, more generally, the $n$th level stabilizer $\operatorname{st}(n)$ is the subgroup of Aut $\mathcal{T}_{d}$ that fixes every vertex of $\mathcal{L}_{n}$. If $G \leq$ Aut $\mathcal{T}_{d}$, we define the $n$th level stabilizer of $G$ as $\operatorname{st}_{G}(n)=\operatorname{st}(n) \cap G$. Notice that stabilizers are normal subgroups of the corresponding group. We let $\psi$ be the isomorphism

$$
\begin{aligned}
\psi: \operatorname{st}(1) & \longrightarrow \operatorname{Aut} \mathcal{T}_{d} \times \cdots \times \text { Aut } \mathcal{T}_{d} \\
g & \longmapsto\left(g_{u}\right)_{u \in \mathcal{L}_{1}},
\end{aligned}
$$

where $g_{u}$ is the section of $g$ at the vertex $u$, i.e. the action of $g$ on the subtree $\mathcal{T}_{u}$ that hangs from the vertex $u$. Let $S_{d}$ be the symmetric group on $d$ letters. An automorphism $a \in$ Aut $\mathcal{T}_{d}$ is called rooted if there exists a permutation $\sigma \in S_{d}$ such that $a$ permutes rigidly the vertices of the subtrees hanging from the first level of the tree according to the permutation $\sigma$, i.e. if $v=x u \in V\left(\mathcal{T}_{d}\right)$, with $x \in \mathcal{L}_{1}$, then $a(x u)=\sigma(x) u$. We usually identify $a$ and $\sigma$. 
Notice that if $g \in \operatorname{st}(1)$ with $\psi(g)=\left(g_{1}, \ldots, g_{d}\right)$, and $\sigma$ is a rooted automorphism, then,

$$
\psi\left(g^{\sigma}\right)=\left(g_{\sigma^{-1}(1)}, \ldots, g_{\sigma^{-1}(d)}\right) .
$$

Any element $g \in G$ can be written uniquely in the form $g=h \sigma$, where $h \in \operatorname{st}(1)$ and $\sigma$ is a rooted automorphism.

Notice also that the decomposition $g=h \sigma$, together with the action (2.1), yields isomorphisms

$$
\begin{aligned}
\text { Aut } \mathcal{T}_{d} & \cong \operatorname{st}(1) \rtimes S_{d} \cong\left(\operatorname{Aut} \mathcal{T}_{d} \times . d \times \text { Aut } \mathcal{T}_{d}\right) \rtimes S_{d} \\
& \cong \operatorname{Aut} \mathcal{T}_{d} \prec S_{d} \cong\left(\left(\cdots>S_{d}\right) \prec S_{d}\right) \prec S_{d} .
\end{aligned}
$$

Throughout the paper, we will use the following shorthand notation: let $f \in$ Aut $\mathcal{T}$ of the form $f=g h$, where $g \in \operatorname{st}_{G}(1)$ and $h$ is the rooted automorphism corresponding to the permutation $\sigma \in S_{d}$. If $\psi(g)=\left(g_{1}, \ldots, g_{d}\right)$, we write $f=\left(g_{1}, \ldots, g_{d}\right) \sigma$.

Definition 2.1. Let $G \leq$ Aut $\mathcal{T}_{d}$, and let $V\left(\mathcal{T}_{d}\right)$ be the set of vertices of $\mathcal{T}_{d}$. Then:

(a) The group $G$ is said to be self-similar if for any $g \in G$ we have

$$
\left\{g_{u} \mid g \in G, u \in V\left(\mathcal{T}_{d}\right)\right\} \subseteq G .
$$

In other words, the sections of $g$ at any vertex are still elements of $G$. For example, Aut $\mathcal{T}_{d}$ is self-similar.

(b) A self-similar group $G$ is said to be fractal if $\psi_{u}\left(\operatorname{st}_{G}(u)\right)=G$ for all $u \in V\left(\mathcal{T}_{d}\right)$, where $\psi_{u}$ is the homomorphism sending $g \in \operatorname{st}(u)$ to its section $g_{u}$.

To prove that a group is self-similar it suffices to show that the condition above is satisfied by the vertices of the first level of the tree (see [3, Proposition 3.1]). The situation is similar in the case of fractal groups. More precisely, using Lemma 2.2, we deduce that to show that a group $G$ is fractal, it is enough to check the vertices in the first level of $\mathcal{T}_{d}$. We recall that $G$ is said to be level transitive if it acts transitively on every level of the tree.

Lemma 2.2 ([11, Lemma 2.7]). If $G \leq$ Aut $\mathcal{T}_{d}$ is transitive on the first level and $\psi_{x}\left(\operatorname{st}_{G}(x)\right)=G$ for some $x \in \mathcal{L}_{1}$, then $G$ is fractal and level transitive.

Here we present a family of non-contracting weakly branch groups. To this end, in the following, we recall the corresponding two definitions.

Definition 2.3. A self-similar group $G \leq$ Aut $\mathcal{T}_{d}$ is contracting if there exists a finite subset $\mathcal{F} \subseteq G$ such that for every $g \in G$ there is $n$ such that $g_{v}$ belongs to $\mathcal{F}$ for all vertices $v$ of $\mathcal{L}_{\geq n}$. Note that if you take two finite sets $\mathcal{F}_{1}$ and $\mathcal{F}_{2}$ satisfying the condition on the sections above, then also $\mathcal{F}_{1} \cap \mathcal{F}_{2}$ will satisfy the condition. For this reason, one can consider the set that is intersection of such sets. This is called the nucleus of $G$ and it is denoted by $\mathcal{N}$.

Definition 2.4. Let $G$ be a self-similar subgroup of Aut $\mathcal{T}_{d}$. We say that $G$ is weakly regular branch over a subgroup $K \leq G$ if $G$ is level transitive and we have

$$
\psi\left(K \cap \operatorname{st}_{G}(1)\right) \geq K \times \cdots \times K .
$$

If, additionally, $K$ is of finite index in $G$, then $G$ is said to be regular branch over $K$. 


\section{The groups $\mathcal{M}(d)$}

Let $d \geq 2$, and let $\mathcal{T}_{d}$ be the $d$-adic tree. The group $\mathcal{M}(d) \leq$ Aut $\mathcal{T}_{d}$ is generated by $d$ elements $m_{1}, \ldots, m_{d}$, where $m_{1}, \ldots, m_{d}$ are defined recursively as follows:

$$
\begin{aligned}
m_{1} & =\left(1, \ldots, 1, m_{1}\right)(1 \ldots d) \\
m_{2} & =\left(1, \ldots, 1, m_{2}, 1\right)(1 \ldots d-1) \\
m_{3} & =\left(1, \ldots, m_{3}, 1,1\right)(1 \ldots d-2) \\
& \vdots \\
m_{d-1} & =\left(1, m_{d-1}, 1, \ldots, 1\right)(12) \\
m_{d} & =\left(m_{1}, \ldots, m_{d}\right) .
\end{aligned}
$$

For example, for $d=3$, we have $\mathcal{M}(3)=\left\langle m_{1}, m_{2}, m_{3}\right\rangle$, where

$$
m_{1}=\left(1,1, m_{1}\right)(123), \quad m_{2}=\left(1, m_{2}, 1\right)(12), \quad m_{3}=\left(m_{1}, m_{2}, m_{3}\right) .
$$

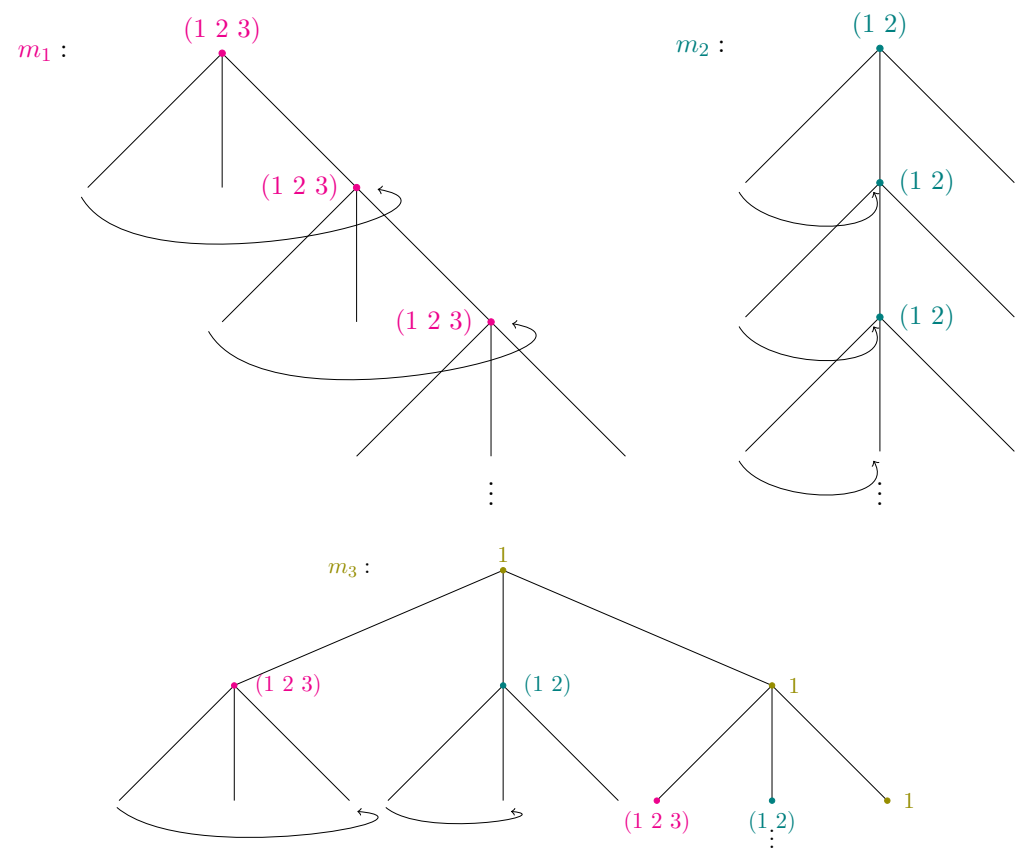

Figure 1: The generators of $\mathcal{M}(3)$.

\subsection{Proof of the main theorem}

In this section we prove the main result of the paper. In order to ease notation, and unless it is strictly necessary, we will simply write $\mathcal{M}$ to denote an arbitrary group $\mathcal{M}(d)$.

Proposition 3.1. The group $\mathcal{M}$ is fractal and level transitive. 
Proof. Notice that the group is transitive on the first level because the rooted part of the generator $m_{1}$ is $(12 \ldots d)$. Also, it is straightforward to see that the group is self-similar, since the sections of every generator at the first level are generators of $\mathcal{M}$. To see that $\mathcal{M}$ is fractal, note that

$$
\begin{aligned}
m_{1}^{d} & =\left(m_{1}, \ldots, m_{1}\right) \\
m_{d}^{m_{1}^{d-2}} & =\left(m_{3}^{m_{1}}, \ldots, m_{2}\right) \\
& \vdots \\
m_{d}^{m_{1}^{2}} & =\left(m_{d-1}^{m_{1}}, \ldots, m_{d-2}\right) \\
m_{d}^{m_{1}} & =\left(m_{d}^{m_{1}}, \ldots, m_{d-1}\right) \\
m_{d} & =\left(m_{1}, \ldots, m_{d}\right) .
\end{aligned}
$$

Then in the last component of the elements above we obtain all the generators of $\mathcal{M}$. Using Lemma 2.2, we conclude that $\mathcal{M}$ is level transitive and fractal.

Proposition 3.2. Let $d \geq 2$. Then the group $\mathcal{M}(d)$ is weakly regular branch over its derived subgroup $\mathcal{M}^{\prime}(d)$.

Proof. We will distinguish the case $d=2$, and $d \geq 3$ separately. Let $d=2$. The element $\left[m_{1}, m_{2}\right]$ is non-trivial since

$$
\left[m_{1}, m_{2}\right]=\left(m_{1}^{-1} m_{2}^{-1} m_{1}^{2}, m_{1}^{-1} m_{2}\right),
$$

and $m_{1}^{-1} m_{2} \notin \operatorname{st} \mathcal{M}(1)$. Then $\mathcal{M}(2)^{\prime}$ is non-trivial, and we have

$$
\left[m_{1}^{2}, m_{2}\right]=\left(1,\left[m_{1}, m_{2}\right]\right) .
$$

From (3.1) and since $\mathcal{M}(2)^{\prime}=\left\langle\left[m_{1}, m_{2}\right]\right\rangle^{\mathcal{N}(2)}$, we obtain that $\{1\} \times \mathcal{M}(2)^{\prime} \leq \psi\left(\mathcal{M}(2)^{\prime}\right)$. As $\mathcal{M}(2)$ is level transitive, we conclude that $\mathcal{M}(2)^{\prime} \times \mathcal{M}(2)^{\prime} \leq \psi\left(\mathcal{M}(2)^{\prime}\right)$, as desired.

Let $d \geq 3$, and write $\mathcal{M}$ for $\mathcal{M}(d)$. First we show that $\mathcal{M}^{\prime}$ is non-trivial. Let us denote $\sigma=\left(\begin{array}{lll}1 & 2 \ldots d\end{array}\right)$ and $\tau=\left(\begin{array}{llll}1 & 2 \ldots d-1\end{array}\right)$. We have

$$
\begin{aligned}
{\left[m_{1}, m_{2}\right] } & =\sigma^{-1}\left(1, \ldots, 1, m_{1}^{-1}\right) \tau^{-1}\left(1, \ldots, 1, m_{2}^{-1}, m_{1}\right) \sigma\left(1, \ldots, 1, m_{2}, 1\right) \tau \\
& =\left(1, \ldots, 1, m_{1}^{-1}\right)^{\sigma}\left(1, \ldots, 1, m_{2}^{-1}, m_{1}\right)^{\tau \sigma}\left(1, \ldots, 1, m_{2}, 1\right)^{\tau^{\sigma}}[\sigma, \tau] \\
& =\left(m_{1}^{-1}, 1, \ldots, 1\right)\left(m_{1}, m_{2}^{-1}, 1, \ldots, 1\right)\left(1, \ldots, 1, m_{2}\right)(12 d) .
\end{aligned}
$$

Hence, we obtain that

$$
\left[m_{1}, m_{2}\right]=\left(1, m_{2}^{-1}, 1, \ldots, 1, m_{2}\right)(12 d) .
$$

By (3.2), we have $\left[m_{1}, m_{2}\right] \notin \operatorname{st}_{\mathcal{M}}(1)$, thus $\mathcal{M}^{\prime}$ is non-trivial.

Now, for $i=1, \ldots, d-2$, and $j=i+1, \ldots, d-1$, we have

$$
\left[m_{i}^{d+1-i}, m_{j}\right]^{m_{1}^{d-1}}=\left(1, \ldots, 1,\left[m_{i}, m_{j}\right]\right) .
$$

Then in order to prove that $\{1\} \times \cdots \times\{1\} \times \mathcal{M}^{\prime} \leq \psi\left(\mathcal{M}^{\prime} \cap \operatorname{st} \mathcal{M}(1)\right)$, it only remains to show that for any $i=1, \ldots, d-1$, there exists $x(i) \in \mathcal{M}^{\prime} \cap \operatorname{st}_{\mathcal{M}}(1)$ such that

$$
x(i)=\left(1, \ldots, 1,\left[m_{i}, m_{d}\right]\right) .
$$


To find such $x(i)$, we first observe that

$$
\left[\left(m_{i}^{d+1-i}\right)^{m_{1}^{i-1}}, m_{d}\right]=\left(1, .{ }^{i} ., 1,\left[m_{i}, m_{i+1}\right], \ldots,\left[m_{i}, m_{d-1}\right],\left[m_{i}, m_{d}\right]\right) .
$$

In order to cancel all these commutators above except for the last component, we use (3.3), and we observe that since $\mathcal{M}$ is level transitive, if we conjugate with a suitable power of $m_{1}$, we get $\left[m_{i}, m_{i+1}\right]^{-1}, \ldots,\left[m_{i}, m_{d-1}\right]^{-1}$ in each component. For example, if $i=2$, we have

$$
\left[\left(m_{2}^{d-1}\right)^{m_{1}}, m_{d}\right]=\left(1,1,\left[m_{2}, m_{3}\right],\left[m_{2}, m_{4}\right], \ldots,\left[m_{2}, m_{d}\right]\right) .
$$

By using the considerations above, we obtain that $x(2)$ must be of the form

$$
\begin{aligned}
x(2) & =\left[m_{3}, m_{2}^{d-1}\right]^{m_{1}^{2}}\left[m_{4}, m_{2}^{d-1}\right]^{m_{1}^{3}} \ldots\left[m_{d-1}, m_{2}^{d-1}\right]^{m_{1}^{d-2}}\left[\left(m_{2}^{d-1}\right)^{m_{1}}, m_{d}\right] \\
& =\left(1, \ldots, 1,\left[m_{2}, m_{d}\right]\right) .
\end{aligned}
$$

To prove last part of the main theorem (that $\mathcal{M}(d)$ is non-contracting), we need some preliminary tools. Namely, we show some results regarding the order of elements of $\mathcal{M}(d)$. We will handle the case $d=2$, and $d>2$ separately. More precisely, we first prove that $\mathcal{M}(2)$ is torsion-free, and then, for $d>2$, we show that the groups $\mathcal{M}(d)$ are neither torsion-free nor torsion, contrary to the case $d=2$.

The following Remark 3.3 and Lemma 3.4 are key steps to prove that $\mathcal{M}(2)$ is torsionfree. We write $\mathcal{M}$ for $\mathcal{M}(2)$.

Remark 3.3. Let $h \in \mathcal{M}^{\prime}$ with $h=\left(h_{1}, h_{2}\right)$. Then $h_{1} h_{2} \in \mathcal{M}^{\prime}$.

Proof. Consider the following map $\rho$ :

$$
\begin{aligned}
\rho: \operatorname{st}_{\mathcal{M}}(1) & \rightarrow \mathcal{M} \quad \rightarrow \mathcal{M} / \mathcal{M}^{\prime} \\
\left(h_{1}, h_{2}\right) & \mapsto h_{1} h_{2} \mapsto \overline{h_{1} h_{2}} .
\end{aligned}
$$

Note that $\rho$ is a homomorphism of groups since $\mathcal{M} / \mathcal{M}^{\prime}$ is abelian. As $\operatorname{st}_{\mathcal{M}}(1) / \operatorname{Ker} \rho$ is abelian, $\mathcal{M}^{\prime} \leq \operatorname{Ker} \rho$. This concludes the proof.

In the proof of next lemma, for a prime $p$ we denote with $\nu_{p}(m)$ the $p$-adic valuation of $m$, that is the highest power of $p$ that divides $m$.

Lemma 3.4. We have $\mathcal{M}^{\prime}=\left(\mathcal{M}^{\prime} \times \mathcal{M}^{\prime}\right)\left\langle\left[m_{1}, m_{2}\right]\right\rangle$. Furthermore

$$
\mathcal{M} / \mathcal{M}^{\prime} \cong\left\langle m_{1} \mathcal{M}\right\rangle \times\left\langle m_{2} \mathcal{M}\right\rangle \cong \mathbb{Z} \times \mathbb{Z}
$$

Proof. Since $\mathcal{M}$ is weakly regular branch over $\mathcal{M}^{\prime}$ by Proposition 3.2, and

$$
\left[m_{1}, m_{2}\right]=\left(\left[m_{1}, m_{2}\right] m_{2}^{-1} m_{1}, m_{1}^{-1} m_{2}\right),
$$

we deduce that $\left(m_{2}^{-1} m_{1}, m_{1}^{-1} m_{2}\right)$ is an element of $\mathcal{M}^{\prime}$. Furthermore, we claim that the elements $\left[m_{1}, m_{2}\right]^{y}$ where $y \in\left\{m_{1}, m_{2}, m_{1}^{-1}, m_{2}^{-1}\right\}$ are in $\left\langle\left[m_{1}, m_{2}\right]\right\rangle$ modulo $\mathcal{M}^{\prime} \times \mathcal{M}^{\prime}$. Indeed, we have

$$
\begin{aligned}
{\left[m_{1}, m_{2}\right]^{m_{1}} } & =\left(m_{1}{ }^{-2} m_{2} m_{1}, m_{1}{ }^{-1} m_{2}^{-1} m_{1}{ }^{2}\right) \\
& =\left(\left[m_{1}{ }^{2}, m_{2}{ }^{-1}\right] m_{2} m_{1}{ }^{-1},\left[m_{1}, m_{2}\right] m_{2}{ }^{-1} m_{1}\right) \\
& \equiv\left(m_{1}{ }^{-1} m_{2}, m_{2}{ }^{-1} m_{1}\right) \quad\left(\bmod \mathcal{M}^{\prime} \times \mathcal{M}^{\prime}\right)
\end{aligned}
$$


and similarly for the other commutators. Thus $\mathcal{M}^{\prime}=\left(\mathcal{M}^{\prime} \times \mathcal{M}^{\prime}\right)\left\langle\left[m_{1}, m_{2}\right]\right\rangle$, as required.

Now we claim that $m_{1}$ is of infinite order. By way of contradiction suppose that, for some $k, m_{1}$ has order $n=2 k$, as $m_{1}$ has order 2 modulo the first level stabilizer. We have

$$
m_{1}^{n}=\left(m_{1}^{k}, m_{1}^{k}\right)=(1,1),
$$

which yields a contradiction as $k<n$. This concludes the proof of the claim and implies that also $m_{2}$ is of infinite order, since $m_{2}=\left(m_{1}, m_{2}\right)$.

Now we want to show that if $m_{1}^{i} m_{2}^{j} \in \mathcal{M}^{\prime}$, then necessarily $i=j=0$. As $m_{1}^{i} m_{2}^{j} \in$ $\mathcal{M}^{\prime} \leq \operatorname{st}_{\mathcal{M}}(1)$, then $i$ must be even. By way of contradiction, we choose the element $m_{1}^{i} m_{2}^{j} \in \mathcal{M}^{\prime}$ subject to the condition that $i$ is divisible by the least possible positive power of 2 , say $2^{a}$, for some $a$. In other words, $\nu_{2}(i)=a$. Then if $m_{1}^{r} m_{2}^{s} \in \mathcal{M}^{\prime}$, necessarily $2^{a} \mid r$. Note that it cannot happen that $r=0$ and $s \neq 0$ as $m_{2}$ is of infinite order. Now, writing $i=2 i_{1}$ for some $i_{1}$, we have

$m_{1}^{i} m_{2}^{j}=\left(m_{1}{ }^{i_{1}+j}, m_{1}{ }^{i_{1}} m_{2}{ }^{j}\right) \equiv\left[m_{1}^{k}, m_{2}^{k}\right] \equiv\left(m_{1}{ }^{k} m_{2}{ }^{-k}, m_{1}{ }^{-k} m_{2}{ }^{k}\right) \quad\left(\bmod \mathcal{M}^{\prime} \times \mathcal{M}^{\prime}\right)$.

This implies that $m_{1}{ }^{i_{1}+j-k} m_{2}{ }^{k} \in \mathcal{M}^{\prime}$ and $m_{1}{ }^{i_{1}+k} m_{2}{ }^{j-k} \in \mathcal{M}^{\prime}$. As $2^{a} \mid i_{1}+j-k$ and $2^{a} \mid i_{1}+k$, then $2^{a}$ divides also $j$. This is because $2^{a} \mid 2 i_{1}+j=i+j$ and by hypothesis $2^{a} \mid i$. Finally, we also have $m_{1}{ }^{i_{1}+k} m_{2}{ }^{j-k} \in \mathcal{M}^{\prime}$, from which we get

$$
m_{1}{ }^{i_{1}+k} m_{2}{ }^{j-k}=\left(m_{1}^{\frac{i_{1}+k}{2}+j-k}, m_{1}^{\frac{i_{1}+k}{2}} m_{2}^{j-k}\right) .
$$

By Remark 3.3, we have $m_{1}^{i_{1}+j} m_{2}^{j-k} \in \mathcal{M}^{\prime}$ which implies that $2^{a} \mid i_{1}+j$. As $\nu_{2}\left(i_{1}\right)=$ $a-1$ and $2^{a} \mid j$, then $\nu_{2}\left(i_{1}+j\right)=a-1$, a contradiction as $2^{a} \mid i_{1}+j$. This completes the proof.

As a consequence, we prove the following.

Proposition 3.5. The group $\mathcal{M}(2)$ is torsion-free.

Proof. Suppose by way of contradiction that there exists an element of finite order in $\mathcal{M}$. Since $\mathcal{M} / \mathcal{M}^{\prime} \cong \mathbb{Z} \times \mathbb{Z}$ by Lemma 3.4 , then this element must lie in $\mathcal{M}^{\prime} \leq \operatorname{st}_{\mathcal{M}}(1)$. Suppose that among all elements of finite order, we take the element $g$ that lies in $\operatorname{st}_{\mathcal{M}}(n) \backslash \operatorname{st}_{\mathcal{M}}(n+1)$, with $n$ minimum with this property. Write $g=\left(g_{1}, g_{2}\right)$. As $g$ is of finite order, then also $g_{1}, g_{2}$ must be of finite order. By our minimality assumption of $n$, the elements $g_{1}, g_{2}$ must lie at least in $\operatorname{st}_{\mathcal{M}}(n)$. This implies that $g=\left(g_{1}, g_{2}\right) \in \operatorname{st}_{\mathcal{M}}(n+1)$, a contradiction to the fact that $g \in \operatorname{st}_{\mathcal{M}}(n) \backslash \operatorname{st}_{\mathcal{M}}(n+1)$.

In the following we determine the order of some elements of $\mathcal{M}(d)$, for $d>2$.

Proposition 3.6. Let $d>2$. Then the group $\mathcal{M}(d)$ is neither torsion-free nor torsion.

Proof. For ease of notation we write $\mathcal{M}$ for $\mathcal{M}(d)$. We start by proving that the given generators of $\mathcal{M}$ are of infinite order. Consider $m_{1}$, and suppose by way of contradiction that its order is $n$. Then if $m_{1}^{n}=1$, we obtain that $m_{1}^{n}$ must lie in st $\mathcal{M}(1)$. Also, its order must be a multiple of $d$, say $n=d k$ for some $k$, since $m_{1}$ has order $d$ modulo the first level stabilizer. Since $m_{1}=\left(1, \ldots, 1, m_{1}\right)(12 \ldots d)$, we obtain

$$
m_{1}^{n}=\left(m_{1}^{k}, \ldots, m_{1}^{k}\right)=(1, \ldots, 1) .
$$


This yields a contradiction since $m_{1}^{k}=1$ and $k<n$. Similar arguments can be used for the generators $m_{2}, \ldots, m_{d-1}$, and $m_{d}$ has infinite order because $m_{d}=\left(m_{1}, \ldots, m_{d}\right)$.

Furthermore, by (3.2), we have

$$
\left[m_{1}, m_{2}\right]=\left(1, m_{2}^{-1}, 1, \ldots, 1, m_{2}\right)(12 d) .
$$

Thus it follows readily that $\left[m_{1}, m_{2}\right]^{3}=1$. Hence $\mathcal{M}$ is not torsion-free.

We conclude the paper by proving the remaining part of the main theorem.

Proposition 3.7. The group $\mathcal{M}$ is non-contracting.

Proof. Suppose by way of contradiction that $\mathcal{M}$ is contracting with nucleus $\mathcal{N}$. Notice that the element $m_{d}^{m_{1}}$ stabilizes the vertex 1 . As a consequence, by induction, $m_{d}^{m_{1}}$ fixes all the vertices of the path $v=1 . n .1$ for all $n \geq 1$. Also, $\left(m_{d}^{m_{1}}\right)_{v}=m_{d}^{m_{1}}$. Clearly, this implies that $m_{d}^{m_{1}}$ lies in $\mathcal{N}$. Consider now a power $k$ of $m_{d}^{m_{1}}$. Arguing as before, we obtain again that $\left(m_{d}^{m_{1}}\right)^{k}$ fixes $v$ and its section at $v$ is $\left(m_{d}^{m_{1}}\right)^{k}$. Thus, $\left(m_{d}^{m_{1}}\right)^{k} \in \mathcal{N}$ for any $k \geq 1$. This concludes the proof since $m_{d}^{m_{1}}$ has infinite order.

\section{References}

[1] L. Bartholdi, R. I. Grigorchuk and Z. Šunić, Branch groups, in: M. Hazewinkel (ed.), Handbook of Algebra, Volume 3, North-Holland, Amsterdam, pp. 989-1112, 2003, doi: 10.1016/s1570-7954(03)80078-5.

[2] F. Dahmani, An example of non-contracting weakly branch automaton group, Contemp. Math. 372 (2005), 219-224, doi:10.1090/conm/372/06887.

[3] R. Grigorchuk and Z. Šunić, Self-similarity and branching in group theory, in: C. M. Campbell, M. R. Quick, E. F. Robertson and G. C. Smith (eds.), Groups St. Andrews 2005, Volume 1, Cambridge University Press, Cambridge, volume 339 of London Mathematical Society Lecture Note Series, pp. 36-95, 2007, doi:10.1017/cbo9780511721212.003, Proceedings of the conference held at the University of St. Andrews, St. Andrews, July 30 - August 6, 2005.

[4] R. I. Grigorchuk, Degrees of growth of finitely generated groups, and the theory of invariant means, Math. USSR Izvestiya 25 (1985), 259-300, doi:10.1070/im1985v025n02abeh001281.

[5] R. I. Grigorchuk, On the growth degrees of $p$-groups and torsion-free groups, Math. USSR Sbornik 54 (1986), 185-205, doi:10.1070/sm1986v054n01abeh002967.

[6] N. Gupta and S. Sidki, On the Burnside problem for periodic groups, Math. Z. 182 (1983), 385-388, doi:10.1007/bf01179757.

[7] M. J. Mamaghani, A fractal non-contracting class of automata groups, Bull. Iranian Math. Soc. 29 (2011), 51-64, http: / / bims.iranjournals.ir/article_123.html.

[8] V. Nekrashevych, Self-Similar Groups, volume 117 of Mathematical Surveys and Monographs, American Mathematical Society, 2005, doi:10.1090/surv/117.

[9] S. Sidki and J. Wilson, Free subgroups of branch groups, Arch. Math. (Basel) 80 (2003), 458463, doi:10.1007/s00013-003-0812-2.

[10] R. Skipper, On a Generalization of the Hanoi Towers Group, Ph.D. thesis, Binghamton University, 2018, https://orb.binghamton.edu/dissertation_and_theses/60.

[11] J. Uria-Albizuri, On the concept of fractality for groups of automorphisms of a regular rooted tree, Reports@SCM 2 (2016), 33-44, doi:10.2436/20.2002.02.9. 